\title{
Processamento auditivo em crianças com fissura labiopalatina com e sem história de otite
}

\section{Hearing process in children with cleft lip and palate with or without history of otitis \\ Tamyne Ferreira Duarte de Moraes', Karina Krähembühl Salvador', Mariana Sodário Cruz², Carolina Ferreira Campos ${ }^{3}$, Mariza Ribeiro Feniman'.}

\author{
1) Fonoaudióloga. Mestranda do programa de Pós-Graduação da Faculdade de Odontologia de Bauru - Universidade de São Paulo, área Fonoaudiologia. \\ 2) Mestre. Doutoranda em Saúde Coletiva pela FMB/UNESP \\ 3) Fonoaudióloga Graduada pelo Curso de Fonoaudiologia a FOB/USP. \\ 4) Professora Titular do Departamento de Fonoaudiologia FOB/USP Pesquisa financiada pelo Conselho Nacional de Pesquisas (CNPq) \\ Instituição: Universidade de São Paulo, Faculdade de Odontologia de Bauru, Departamento de Fonoaudiologia, Clínica de Fonoaudiologia \\ Bauru / SP - São Paulo \\ Endereço para correspondência: Mariza Ribeiro Feniman - Alameda Dr. Octávio Pinheiro Brizolla, 9-75 - Vila Universitária - Bauru / SP - Brasil - CEP: $17012-901$ - Telefone: \\ (+55 14) 3235-8332 - E-mail: feniman@usp.br \\ Artigo recebido em 17 de Março de 2011. Artigo aprovado em 21 de Julho de 2011.
}

\section{RESUMO}

Introdução: Considerando que perdas auditivas e complicações otológicas podem interferir no processo de maturação do sistema nervoso central, este estudo teve como objetivo verificar o desempenho de crianças com FLP com e sem histórico de otite média na avaliação do processamento auditivo.

Método: Estudo prospectivo. Foram avaliadas 20 crianças com fissura labiopalatina operada, na faixa etária de 7 a 10 anos, divididas em grupo I - 10 crianças com fissura labiopalatina com história de otite - e grupo II - constituído de 10 crianças com fissura labiopalatina sem história de otite. Foram realizados testes dióticos, monóticos e dicóticos.

Resultados: Todas as crianças apresentaram desempenho ruim em pelo menos um teste dicótico. Nos testes dióticos os maiores índices de desempenho ruim foram das crianças pertencentes ao grupo I, enquanto que nos testes monóticos, o desempenho ruim foi encontrado em maior número nas crianças do grupo II.

Conclusão: Tanto as crianças com história de otite como as sem história de otite apresentaram desempenho ruim nos testes do processamento, ou seja, algum tipo de alteração central foi encontrada em 100\% das crianças estudadas em ambos os grupos.

Palavras-chave: audição, fenda labial, fissura palatina, otite média.

\section{SUMMARY}

Introduction: Considering that hearing loss and otologic complications can interfere in the maturation process of central nervous system, this study aimed to check the performance of children with cleft lip and palate with or without the history of otitis media in the evaluation of the hearing process.

Method: Prospective study. Were evaluated 20 children with operated cleft lip and palate, aged between 7 to 10 years old, divided into group I - 10 children with cleft lip and palate with history of otitis - and group II - with 10 children with cleft lip and palate with no history of otitis. Were performed diotic, monotic and dichotic tests.

Results: All the children presented a bad performance in at least one dichotic test. In these diotic tests, children of group I had the worst performance, while in the monotic tests the worst performance was of group II.

Conclusion: All the children with history of otitis and all the children with no history have presented a bad performance in the processing tests,in other words, some kind of central alteration was found in $100 \%$ of the children studied in both groups.

Keywords: hearing, cleft lip, cleft palate, otitis media. 


\section{INTRODUÇÃO}

Com o objetivo de se obter uma adequada avaliação de todo o sistema vestíbulo-coclear, a utilização de testes que avaliam o processamento auditivo tem se mostrado um instrumento desafiador da audiologia clínica (14).

Perdas auditivas e histórias de otite média podem ser indicadores de risco para o desenvolvimento do processamento auditivo, assim como para o desenvolvimento da linguagem, fala e aprendizagem, visto que a literatura tem demonstrado que crianças com histórico de otites de repetição durante a infância tendem a apresentar diferenças significantes no desempenho nas atividades de percepção auditiva, memória auditiva, a quisição de linguagem oral e progresso escolar $(4,16)$.

Segundo a American Speech Hearing Association (2) (ASHA), distúrbio do processamento auditivo (DPA) é uma alteração em um ou mais mecanismos ou processos do sistema auditivo responsáveis pelos comportamentos de localização e lateralização sonora; discriminação auditiva; reconhecimento de padrões auditivos; aspectos temporais da audição, incluindo resolução, mascaramento, integração e ordenação temporal; desempenho auditivo na presença de sinais competitivos e desempenho auditivo com sinais acústicos degradados.

Ao estudar a relação entre a otite média recorrente, linguagem e processamento auditivo, foram verificados que a linguagem e processamento auditivo de crianças com história de Otite Média com Efusão (OME) apresentaram resultados significantemente piores que crianças que não apresentavam história (8). Diversos autores afirmaram que crianças com otites de repetição durante a infância tendem a apresentar diferenças significantes no desempenho nas atividades de percepção auditiva, memória auditiva, aquisição de linguagem oral e progresso escolar (4, 3, 20,29).

Outros autores (23) que afirmaram que sujeitos com perda auditiva periférica provavelmente terão desempenho prejudicado nos testes do processamento auditivo. No entanto, pode haver pessoas que, mesmo apresentando perda auditiva periférica (de grau leve a moderado), apresentam processamento auditivo normal.

A fissura labiopalatina (FLP) é resultado de uma malformação decorrente de falhas no desenvolvimento ou na maturação dos processos embrionários (1).

Diversos estudos $(10,11,21,27)$ deram ênfase à audição periférica na população com FLP, sendo amplamente conhecida à alta incidência de alterações de orelha média e/ ou perdas de audição, decorrentes de um mecanismo de aeração velotubal deficiente. Assim, a otite média e perdas auditivas do tipo condutivo, geralmente bilaterais, vêm figurando como patologias importantes nessa população. Essas alterações têm como consequência uma privação sensorial, levando a um indicador de risco para alterações no desenvolvimento do processamento auditivo, da linguagem, da fala, aprendizagem e potencial cognitivo da criança com FLP.

Entretanto, estudos mostram-se emergentes $(5,7,9,17,18)$ sobre o processamento auditivo em crianças com FLP.

A identificação de dificuldades do processamento auditivo verbal e não verbal em crianças pré-escolares e escolares tem sua importância afirmada embases acadêmicas, maturacionais, psicológicas e econômicas. A identificação precoce de crianças com limitações nas habilidades de processamento da informação auditiva reduzem o tempo e os custos da intervenção (15).

Considerando que perdas auditivas e complicações otológicas podem interferir no processo de maturação do sistema nervoso central, este estudo teve como objetivo verificar o desempenho de crianças com FLP com e sem histórico de otite média na avaliação do processamento auditivo.

\section{MÉTOdO}

Este trabalho foi aprovado pelo Comitê de Ética em Pesquisa da instituição onde foi realizado, com protocolo número 15/2001.

Participaram deste estudo 20 crianças de ambos os gêneros matriculados regularmente em um hospital de referência em reabilitação de anomalias craniofaciais no interior do estado de São Paulo.

Os critérios de inclusão dos participantes deste estudo foram: ter FLP operada, faixa etária de 7 a 11 anos, não apresentar queixa e/ou infecção das vias aéreas superiores em situação de exame, não apresentar histórico de desordens neurológicas, concordância dos pais e assinatura com o Termo de Consentimento Livre e Esclarecido.

As crianças formaram dois grupos, divididos segundo a presença ou não de história de otite:

- Grupo I (GI) - constituído de 10 crianças com FLP com história de otite.

- Grupo II (GII) - constituído de 10 crianças com FLP sem história de otite.

Foi realizada comparação entre os achados dos grupos amostrados (GI e o GII). 
Os testes do processamento auditivo utilizados foram selecionados considerando a faixa etária e o desenvolvimento da audição.

Os testes aplicados se dividemem dióticos, monóticos e dicóticos.

Os testes dióticos (22) são testes onde estímulos iguais são apresentados simultaneamente para ambas as orelhas. São eles: Teste de Localização Sonora em cinco direções (direita, esquerda, atrás, acima e à frente) (22), Testes de Memória para Sons Verbais e Não-Verbais em Sequência (22) e o Teste da Fusão Auditiva-Revisado-AFT$\mathrm{R}$ (24) que é um procedimento para medir a habilidade do processamento temporal, determinando a duração (em ms) em que o ouvinte pode detectar um breve intervalo de silêncio entre dois tons, e relatar se ele ouviu um ou dois tons.

Os testes monóticos (28) são os testes nos quais estímulos diferentes ou não são apresentados simultaneamente na mesma orelha, ou seja, ipsilateralmente. São eles: Teste de Palavras e Frases com Mensagem Competitiva Ipsilateral - Pediatric Speech Inteligibility (PSI) em que os estímulos verbais utilizados são 10 frases que são apresentadas aleatoriamente, juntamente com a mensagem competitiva, e devem ser identificadas pela criança por meio da indicação da figura que a representa, sendo que a mensagem competitiva é uma história infantil; o Teste de Fala no Ruído com Figuras e com Palavras (PSI com palavras) composto por 10 palavras que devem ser identificadas por meio da figura correspondente à palavra ouvida podendo ser realizado com duas formas distintas de mensagem competitiva ipsilateralmente (o ruído branco ou uma história infantil); o Teste de Frases com Mensagem Competitiva Ipsilateral (SSI) em que são utilizados estímulos verbais compostos por 10 frases sintéticas de terceira ordem e a mensagem competitiva utilizada é um texto de história do Brasil.

Os testes dicóticos são compostos por estímulos diferentes apresentados simultaneamente para ambas as orelhas. O Teste Dicótico com Sons Não-Verbais Competitivos (22) é realizado com três sons ambientais (barulho de trovão, barulho de sino de igreja e barulho de uma porta batendo) e três sons onomatopeicos (som de gato miando, cachorro latindo e galo cacarejando), que devem ser identificados por meio da indicação das figuras que os representam. Estes sons foram combinados entre si e sincronizados no tempo a fim de formar doze pares. No Teste Sttagered Spondaic Word (SSW) (6) são utilizados como estímulos verbais 160 palavras dissílabas compostas do português brasileiro e o Teste Dicótico de Dígitos (26) são constituído por 20 pares de dígitos que representam dissílabos na língua portuguesa.

Estes testes foram realizados em cabina acústica por meio de um audiômetro de dois canais acoplado a um CD player.

Os resultados foram analisados e comparados com o padrão de normalidade proposto por cada teste, sendo classificados como desempenho ruim os que apresentaram escores abaixo da normalidade e desempenho bom os demais.

Foi realizada análise estatística descritiva dos grupos de acordo com o desempenho em cada teste, comparando os resultados do grupo I e grupo II.

\section{RESULTADOS}

Algumas crianças que participaram deste estudo não puderam realizar todos os testes propostos e descritos na metodologia deste trabalho, pois não compreenderam as instruções para realização do teste ou apresentavam problemas articulatórios. Assim, o teste Dicótico de Dígitos não foi realizado em uma criança (GI), o teste SSW não foi realizado em quatro crianças (1 GI e 3 GII) e o teste SSI/ PSI não foi realizado em duas crianças (GII).

Na Tabela 1 estão descritos os resultados dos testes

Tabela I . Descrição dos resultados dos testes avaliados.

\begin{tabular}{|c|c|c|c|c|c|c|}
\hline & \multicolumn{2}{|c|}{ Desempenho ruim } & \multicolumn{2}{|c|}{ Desempenhobom } & \multicolumn{2}{|c|}{ Total } \\
\hline & Gl & Gll & Gl & Gll & Gl & Gll \\
\hline $\begin{array}{l}\text { Testes dióticos } \\
\text { Localização } \\
\text { Memória } \\
\text { AFT-R }\end{array}$ & $\begin{array}{l}\% \text { (N) } \\
30 \%(3) \\
30 \%(3) \\
60 \%(6)\end{array}$ & $\begin{array}{l}\%(\mathrm{~N}) \\
10 \%(\mathrm{I}) \\
40 \%(4) \\
40 \%(4)\end{array}$ & $\begin{array}{l}\%(\mathrm{~N}) \\
70 \%(7) \\
70 \%(7) \\
40 \%(4)\end{array}$ & $\begin{array}{l}\text { \% (N) } \\
90 \%(9) \\
60 \%(6) \\
60 \%(6)\end{array}$ & $\begin{array}{l}\% \quad(\mathrm{~N}) \\
100 \%(10) \\
100 \%(10) \\
100 \%(10)\end{array}$ & $\begin{array}{l}\%(\mathrm{~N}) \\
100 \%(10) \\
100 \%(10) \\
100 \%(10)\end{array}$ \\
\hline $\begin{array}{l}\text { Testes monóticos } \\
\text { PSI/SSI }\end{array}$ & $60 \%(6)$ & $75 \%(6)$ & $40 \%(4)$ & $25 \%(2)$ & $100 \%(10)$ & $100 \%(8)$ \\
\hline $\begin{array}{l}\text { Testes dicóticos } \\
\text { Sons não verbais } \\
\text { SSW } \\
\text { Dígitos }\end{array}$ & $\begin{array}{c}100 \%(10) \\
89 \%(8) \\
44 \%(4)\end{array}$ & $\begin{array}{c}100 \%(10) \\
85 \%(6) \\
50 \%(5)\end{array}$ & $\begin{array}{l}0 \%(0) \\
11 \%(1) \\
66 \%(5)\end{array}$ & $\begin{array}{c}0 \%(0) \\
15 \%(1) \\
50 \%(5)\end{array}$ & $\begin{array}{c}100 \%(10) \\
100 \%(9) \\
100 \%(9)\end{array}$ & $\begin{array}{c}100 \%(10) \\
100 \%(7) \\
100 \%(10)\end{array}$ \\
\hline
\end{tabular}


avaliados de acordo com o desempenho das crianças nos grupos amostrados.

\section{DISCUSSÃO}

Os dois grupos (GI e GII) apresentaram desempenho ruim na maioria dos testes. Entretanto, a população com história de otite (GI) apresentou resultados piores na avaliação do processamento auditivo, se comparado com a população sem história de otite (GII).

Foi observada que, independente da presença de história de otite nos primeiros anos de vida, a população com fissura labiopalatina apresentou dificuldades consideráveis nos testes aplicados, indicando porcentagem de alteração em ambos os grupos, sendo a otite um agravante desta situação.

Crianças sem história de otite (GII) apresentaram desempenho normal nos testes dióticos, enquanto que, nos demais testes, foi predominante desempenho ruim. Já as crianças com história de otite (GI) apresentaram índices inferiores de desempenho normal, comparadas com o GII, sendo mais frequente o desempenho ruim nos testes dicóticos.

Estes dados estão concordantes com a literatura encontrada, tendo em vista que na maioria dos estudos que envolvem processamento auditivo e FLP os resultados das avaliações apresentam altos índices de alterações. $(7,5)$

Comparando o desempenho bom e ruim entre as crianças avaliadas e pertencentes a um mesmo grupo, foi possível concluir que os testes que apresentaram maiores resultados de desempenho ruim foram o SSW e o Dicótico Não Verbal para GI. No GII, os testes PSI e SSI também apresentaram alto índice de desempenho ruim, além do SSW e Dicótico Não Verbal.

Em crianças sem FLP e com suspeita de alteração auditiva houve maior alteração nos resultados dos testes dicóticos, especialmente o Dicótico de Dígitos e o SSW, sendo que o alto grau linguístico destes testes fez com que fossem mais sensíveis para identificar alterações de processamento auditivo (12).

Com relação ao teste AFT-R, o índice de desempenho alterado do grupo com história de otite (GI - 60\%) foi maior. Sabe-se que um maior limiar de fusão auditiva leva a uma maior probabilidade de déficit de processamento temporal e interferência na percepção de fala (19).

A literatura relata que crianças com FLP apresentam limiares de fusão auditiva significantemente maiores do que crianças sem esta malformação (9). Este fato pode ser justificado pela alta ocorrência de otite na população com FLP.

Presença de história de otite durante a infância pode interferir no desenvolvimento de habilidades auditivas e na percepção de traços distintivos da fala, podendo levar a alterações fonológicas.

Em crianças sem FLP, mas com presença de histórico de otite, os testes monóticos podem apresentar resultados inferiores, quando comparados com os resultados de crianças sem histórico de otite (13).

A presença de histórico de otite pode acarretar em maiores prejuízos nas habilidades auditivas de figura-fundo e fechamento auditivo, memória auditiva e linguagem (25).

A determinação exata das relações entre a otite média e o Transtorno do Processamento Auditivo ainda não foi amplamente elucidada. É necessário cautela nas interpretações dos resultados e determinação precisa de outras variáveis que podem estar intervindo paralelamente como etiologia para o Transtorno do Processamento Auditivo, como desnutrição, ambientes socioculturais pouco estimuladores, redução da motivação e estados de saúde em geral (30).

\section{CONCLUSÃO}

Foi observada que, independente da presença de história de otite nos primeiros anos de vida, a população com FLP apresentou importantes dificuldades nos testes aplicados, indicando porcentagem de desempenho ruim em ambos os grupos, sendo a otite um agravante desta situação.

Desse modo, sugere-se a avaliação do processamento auditivo em toda a população com FLP e não apenas naquela em que as otites são indicadores de risco, a fim de que se estabeleça um amplo e completo trabalho de reabilitação, visto que dificuldades de processamento auditivo podem interferir no desenvolvimento da linguagem e rendimento escolar.

\section{REFERÊNCIAS BIBLIOGRÁFICAS}

1. Altmann EBC. Fissuras labiopalatinas. Carapicuíba: PróFono; 1997.

2. American Speech-language-hearing Association (ASHA) - Central auditory processing: current status of research and implications for clinical practice. Am J Audiol. 1996, 5(2):4154. 
3. Asbjornsen A, Holmefjord A, Reisæeter S, Moller P, Klausen O, Prytz B et al. Lasting auditory attention impairment after persistent middle ear infections: a dichotic listening study. Develop Med Child Neurol. 2000, 42(7):481-486.

4. Balbani APS, Montovani JC. Impacto das otites médias na aquisição da linguagem em crianças. J Pediatr. 2003, 79(5):391-6.

5. Beloni M, Santos MFC. Processamento auditivo em crianças com fissura labiopalatina não-sindrômica. In: Anais do $20^{\circ}$ Encontro Internacional de Audiologia; 2005 abril: São Paulo, SP [em CD-ROM].

6. Borges ACLC. Dissílabos Alternados-SSW. In: Pereira LD, Schochat E. Processamento auditivo central: manual de avaliação. São Paulo: Lovise, p.169-178.

7. Boscariol M, André KD, Feniman MR. Crianças com fissura isolada de palato: desempenho nos testes de processamento auditivo. Braz J Otorhinolaryngol. 2009, 75(2):213-20.

8. Campbell N, Hugo R, Uys I, Hanekom J, Millard S. Early recurrent otitis media, language and central auditory processing in children. S Afr J Commun Disord. 1995, 42:7384

9. Cassab TV, Zorzetto NL. Teste da fusão auditiva-revisado (AFT-R) em crianças com fissura labiopalatina. ACTA ORL/ Técnicas em Otorrinolaringologia. 2006, 24(4):272-76.

10. Feniman MR, Souza AG, Jorge JC, Lauris JRP. Achados otoscópicos e timpanométricos em lactentes com fissura labiopalatina. Rev Bras Otorrinolaringol. 2008, 74(2):24852.

11. Gloudy S, LottD, Canady J, Smith RJH. Conductive hearing loss and otopathology in cleft palate patients. Otolaryngology-Head and Neck Sugery. 2006,134(6):946948.

12. Gonçales AS, Souza LB, Souza VMC. Avaliaçao do processamento auditivo:relato de experiência clínica. In: Aquino AMCM. Processamento Auditivo: eletrofisiologia e pscioacústica. São Paulo: Lovise, 2002.

13. Hall JW, Grose JH. Effect of otitis media witheffusion comodulation masking release in children. J Speech Hear Res. 1994, 37(6):1441-49.

14. Jacob LCB, Alvarenga KF, Zeigelboim BS. Avaliação audiológica do sistema nervoso auditivo central. Arq Int Otorrinolaringol. 2000, 4(4):144-51.

15. Kelly D. A rationale for screening for central auditory processing difficulties at the kindergarten and second-grade levels: considerations for the Speech-Language Pathologist. Disponível em: www.turtan.com.

16. Klausen O, MollerP, Holmefjord A, ReisaeterS, Asbjornsen A. Lasting effects of otitis media with effusion on language skills and listening performance. Acta Otolaryngol Suppl. 2000, 543:73-76.

17. Laasonen M, Haapanen ML, Mäenpää P, Pulkkinen J, Ranta R, Virsu V. Visual, Auditory, and Tactile Temporal Processing in Children With Oral Clefts. Journal of Craniofacial Surgery. 2004, 1(3):510-8.

18. Lemos ICC. Habilidade de Atenção Auditiva em Crianças de Sete Anos Com Fissura Labiopalatina: Estudo Comparativo. Tese, Bauru, 2007.

19. McCrosckey R, Keith RW. AFT-R: Auditory fusion testrevised. San Antonio, TX: Psychological Corporation, 1996.

20. Northern JL, Dows MP. Audição em crianças. São Paulo: Manole, 3 ed., 1989.

21. Pegoraro-Krook MI, Souza JCRD, Teles-Magalhães LC, Feniman MR. Intervenção Fonoaudiológica na Fissura Palatina. In: Ferreira LP, Beffi-Lopes DM, Limongi SCO. Tratado de Fonoaudiologia. São Paulo: Roca. 2004; p. 439455.

22. Pereira LD. Temas Sobre o Desenvolvimento Processamento Auditivo. 1993, (11):7-14.

23. Ramos CS, Pereira LD. Processamento auditivo e audiometria de altas freqüências em escolares de São Paulo. Pró-Fono Rev Atual Cient. 2005, 17(2):153-164.

24. Samelli AG, Schochat E. Processamento auditivo, resolução temporal e teste de detecção de GAP: revisão da literatura. Rev CEFAC. 2008, 10(3):369-377.

25. Santos MFC, ZiliottoKN, Monteiro VG, Hirata CHW, Pereira LD, WeckxLLM. AvaliaçãodoProcessamentoAuditivoCentral em Crianças Com e Sem Antecedentes de Otite Média. Rev Bras Otorrinolaringol. 2001, 67(4):448-54.

26. Santos, MFC. Processamento auditivo central: teste dicótico de dígitos em crianças e adultos normais. Tese, São Paulo, Universidade Federal de São Paulo Escola Paulista de Medicina de Medicina, 1998:198.

27. Souza D, Ninno CQMS, Borges GP, Silva TM, Miranda ES. Perfil audiológico de indivíduos operados de fissura de palato no Hospital da Baleia de Belo Horizonte. ACTA ORL/ Técnicas em Otorrinolaringologia. 2006, 24(3):170-173. 
28. Toniolo IMF, Rossi AG, Borges ACLC, Pereira LD. Processamento Auditivo: habilidade auditiva de memoria sequencial verbal e nao verbal em escolares. Rev Saúde. 1994, 20(3-4):11-22.

29. Wertzner HF, Oliveira MMF. Semelhanças entre sujeitos com distúrbio fonológico. Pró-Fono Revista de Atualização Científica. 2002, 14(2):143-152.
30. Mahon E. Processamento Auditivo Central e Otite Média. 1999. Centro de Especialização em Fonoaudiologia Clínica (CEFAC), Recife. Muscrito. 